04.2

\title{
Перенос тепла и частиц в начальной фазе омических разрядов сферического токамака Глобус-М
}

\author{
() А.Ю. Тельнова, Г.С. Курскиев, Н.Н. Бахарев, В.К. Гусев, Е.О. Киселев, \\ В.Б. Минаев, И.В. Мирошников, М.И. Патров, Ю.В. Петров, \\ Н.В. Сахаров, В.А. Токарев, С.Ю. Толстяков, Е.А. Тюхменева, \\ Н.А. Хромов, П.Б. Щёголев
}

Физико-технический институт им. А.Ф. Иоффе РАН, Санкт-Петербург, Россия

E-mail: anna.telnova@mail.ioffe.ru

Поступило в Редакцию 2 апреля 2019г.

В окончательной редакции 2 апреля 2019 г.

Принято к публикации 4 апреля 2019г.

\begin{abstract}
Представлены результаты экспериментов и моделирования переноса тепла и частиц в сферическом токамаке Глобус-М. Исследования проводились в омическом режиме в водородной и дейтериевой плазме. Показано, что в фазе роста тока при одинаковых начальных условиях до тех пор, пока выполняется условие, при котором минимум запаса устойчивости превышает единицу, возможны два сценария развития разряда: с пикированием электронной температуры либо с пикированием электронной плотности. После того как запас устойчивости достигает значения единицы, различия уже не наблюдаются. Моделирование транспортных процессов с помощью кода ASTRA показало, что пикирование электронной температуры и плотности является следствием снижения переноса тепла и частиц во внутренней части плазменного шнура (т.е. наличия внутренних транспортных барьеров).
\end{abstract}

Ключевые слова: термоядерный синтез, транспортный анализ, омический нагрев плазмы, внутренние транспортные барьеры.

DOI: 10.21883/PJTF.2019.13.47951.17817

На сферическом токамаке Глобус-М (аспектное отношение $A=1.5$, большой радиус $R=0.36 \mathrm{~m}$, малый радиус $a=0.24 \mathrm{~m}$ ) [1] наблюдалось формирование внутренних транспортных барьеров (ВТБ) в разрядах с нейтральной инжекцией в фазе роста тока $[2,3]$. В них ВТБ наблюдался на диффузии $D$ и, как следствие, происходило пикирование электронной плотности. Такой режим существовал до тех пор, пока минимум запаса устойчивости не становился равным единице $\left(q_{\min }=1\right)$. ВТБ в омических разрядах в период $H$-моды также наблюдались [4] и были подробно исследованы [5] на токамаке ТУМАН-3М. В настоящей работе приводятся результаты экспериментов по омическому нагреву плазмы. Особый интерес представляет переходный режим, когда ток плазмы уже достиг своего максимального значения, но $q_{\min }>1$ и еще не появились пилообразные колебания. Данные эксперименты проводились при тороидальном магнитном поле $B_{T}=0.4 \mathrm{~T}$, токе плазмы $I_{p}=200 \mathrm{kA} \mathrm{в}$ диверторной конфигурации с нижней $X$-точкой, вытянутостью $k=1.75$, средней треугольностью $\delta=0.35$ и скоростью роста тока $d I / d t \approx 6.5 \mathrm{MA} / \mathrm{s}$. Менялся только основной рабочий газ: водород или дейтерий. На рис. 1 приведены основные осциллограммы разрядов \#29585 (водород) и \#29819 (дейтерий) из этой серии. Видно, что различия в разрядах появляются после $135 \mathrm{~ms}$, когда в плазме начинается рост мягкого рентгеновского излучения. В разряде \#29585 рост этого излучения начинается только после $140 \mathrm{~ms}$. Пилообразные колебания в разряде \#29819 начинаются раньше. Они выражены сильнее, что является следствием более высокой температуры электронов в центре шнура. Следует отметить, что в разряде \#29585 примерно со 125 по $135 \mathrm{~ms}$ наблюдается значительный всплеск интенсивности светимости линии CIII, что может свидетельствовать о поступлении примесей в плазму. Переход в $H$-моду происходит в обоих разрядах примерно на $135 \mathrm{~ms}$, что видно по падению интенсивности линии $\mathrm{D} \alpha$ на фоне постоянно растущей плотности.

В первом случае (разряд \#29585) сильный градиент образуется на профиле плотности электронов (рис. 2,a), при этом профиль температуры остается плоским (рис. 2,b). Во втором случае (разряд \#29819), наоборот, образуется сильный градиент температуры (рис. 2,d), а профиль плотности плоский (рис. 2,c). Различия сохраняются только при условии $q_{\min }>1$ (т. е. на начальной стадии разряда во время роста тока, рис. 1). При снижении $q_{\min }$ ниже единицы профили сглаживаются (154 ms).

В конце стадии роста тока на $147 \mathrm{~ms}$ в разряде \#29585 фактор пикируемости $\left(n_{e}(0) /\left\langle n_{e}\right\rangle\right)$ достигает 3. В разряде \#29585 отсутствует источник частиц в центре плазмы; следовательно, можно сделать предположение о высоком внутреннем пинче частиц. Также сильный градиент плотности может свидетельствовать о снижении коэффициента диффузии в этой области.

В разряде \#29819 при аналогичных начальных условиях реализуется противоположный сценарий. Профиль плотности плоский, что может свидетельствовать о 


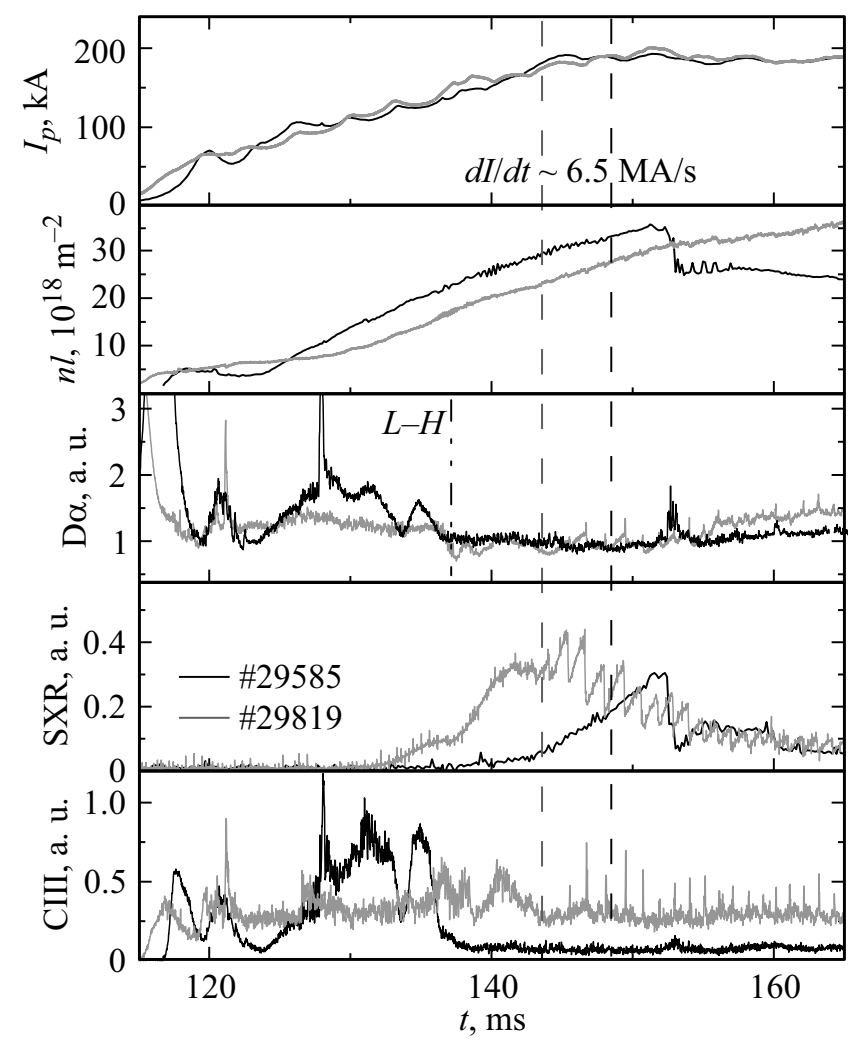

Рис. 1. Временна́я эволюция параметров плазмы: ток плазмы $\left(I_{p}\right)$, среднехордовая плотность $(n l)$, излучение линии $\mathrm{D} \alpha$, сигнал мягкого рентгеновского излучения (SXR), излучение линии CIII. Отмечены момент перехода в $H$-режим и моменты, выбранные для моделирования.

плохом удержании частиц. Однако имеется сильный градиент электронной температуры и предположительно ВТБ на профиле электронной температуры.

Для определения коэффициентов температуропроводности и диффузии в двух рассматриваемых разрядах было проведено моделирование с помощью кода ASTRA [6]. Магнитная конфигурация восстанавливалась на основе данных кода EFIT [7]. Как для электронной температуры, так и для плотности решалась прямая транспортная задача: коэффициенты диффузии и температуропроводности подбирались таким образом, чтобы рассчитанные профили наилучшим образом совпадали с экспериментальными данными. Скорость пинчевания предполагалась вееровской [8] в обоих разрядах. Для ионной температуры решалась прямая транспортная задача на основании неоклассических коэффициентов температуропроводности, так как для сферических токамаков $[9,10]$, в том числе для токамака Глобус-М [11], характерен неоклассический теплоперенос ионов. Рассчитанная ионная температура сравнивалась с измерениями, полученными с помощью анализатора атомов перезарядки АКОРД-12.

Для разряда \#29585 коэффициент электронной температуропроводности предполагался постоянным по про- филю и был рассчитан по формуле [12]:

$$
\chi=k a^{2} /\left(4 \tau_{E}^{\operatorname{IPB} 98(\mathrm{y}, 2)}\right),
$$

где $\tau_{E}^{\mathrm{IPB} 98(\mathrm{y}, 2)}-$ время удержания энергии, рассчитанное по скейлингу ITER Physics Basics 1998 [13]. Коэффициент диффузии подбирался таким образом, чтобы достичь максимального соответствия между экспериментальным профилем плотности электронов и рассчитанным. При этом он подбирался исходя из предположения $D \approx \chi / 2$. В обоих разрядах значение электронной плотности на границе достаточно велико $\left(n_{e} \geqslant 2 \cdot 10^{19} \mathrm{~m}^{-3}\right)$, что является характерной особенностью омического $H$-режима в токамаке Глобус-М. Для учета этого обстоятельства в моделировании на границе плазмы предполагался барьер (порог на коэффициенте диффузии). При подборе коэффициента диффузии выяснилось, что в области относительного малого радиуса $0.3-0.4$ необходимо снизить $D$ до уровня $0.2 \mathrm{~m}^{2} \cdot \mathrm{s}^{-1}$, что близко по величине к уровню диффузии на границе плазмы $\left(D \approx 0.25 \mathrm{~m}^{2} \cdot \mathrm{s}^{-1}\right)$. Это означает, что в разряде присутствует ВТБ (рис. 2,a).

Для разряда \#29819 коэффициенты температуропроводности и диффузии рассчитывались исходя из аналогичных предположений. Расчеты показали, что вблизи $r / a \sim 0.4$ ( $r / a-$ относительный малый радиус) имеется область с коэффициентом электронной температуропроводности, сниженным до уровня $\sim 1 \mathrm{~m}^{2} \cdot \mathrm{s}^{-1}$ (рис. 2,d). Коэффициент диффузии в этом разряде предполагался постоянным $(D \approx \chi / 2)$ со снижением на границе до уровня $D \approx 0.5 \mathrm{~m}^{2} \cdot \mathrm{s}^{-1}$ (что характерно для $H$-моды). Неоклассическая ионная температура соответствует данным, полученным с помощью анализатора атомов перезарядки (экспериментальная $T_{i}=212 \pm 23 \mathrm{eV}$, рассчитанная $\left.T_{i}=230 \mathrm{eV}\right)$.

Таким образом, моделирование показало, что реализуются два возможных сценария развития омического разряда: с формированием ВТБ на электронной температуре либо с формированием ВТБ на электронной плотности. После обработки серии подобных разрядов было обнаружено, что эффект пикирования плотности не зависит от средней плотности плазмы (рис. 3). Объяснением различий в данных разрядах может служить разное количество примесей в плазме (чистота рабочего газа и состояние стенки). Это подтверждается интенсивностью светимости углеродной линии СІІІ в начале водородного разряда (рис. 1). Поступление дополнительной примеси в плазму может значительно влиять на профиль тока, а следовательно, и на профиль запаса устойчивости, магнитный шир и, как следствие, на коэффициенты температуропроводности и диффузии. Более подробный анализ можно провести методом гирокинетического моделирования. Практическую значимость имеют разряды с ВТБ на температуре и высоким коэффициентом диффузии в центре. В них не происходит накопления примесей, и, следовательно, уровень радиационных потерь не слишком высок. Такие сценарии перспективны 

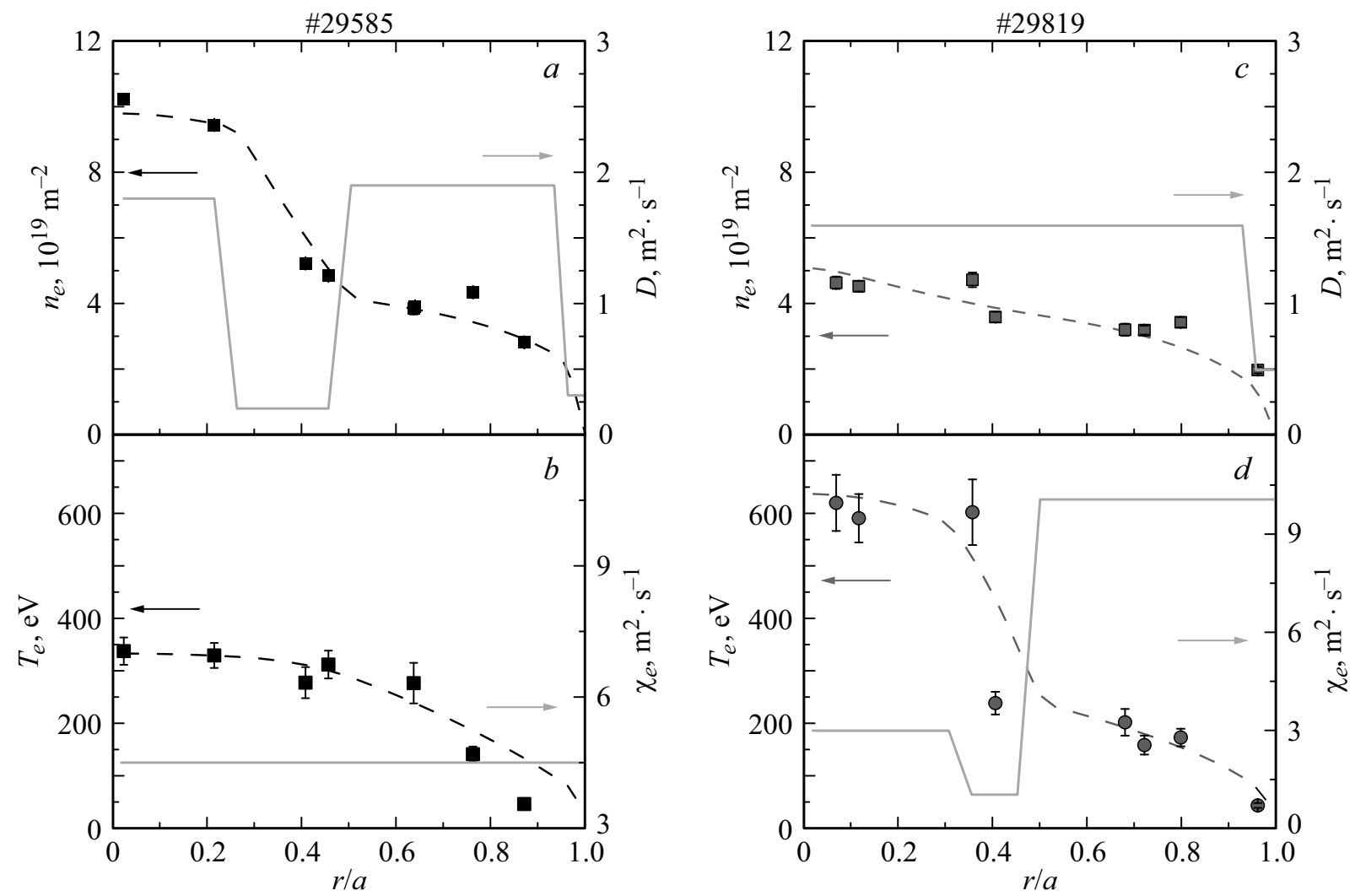

Рис. 2. Распределения в зависимости от относительного малого радиуса $r / a$ экспериментальной и рассчитанной с помощью коэффициента диффузии $D$ электронной плотности $(a, c)$, а также экспериментальной и рассчитанной с помощью коэффициента температуропроводности $\chi_{e}$ электронной температуры $(b, d)$ для разрядов \#29585 $(a, b)$ и $29819(c, d)$.

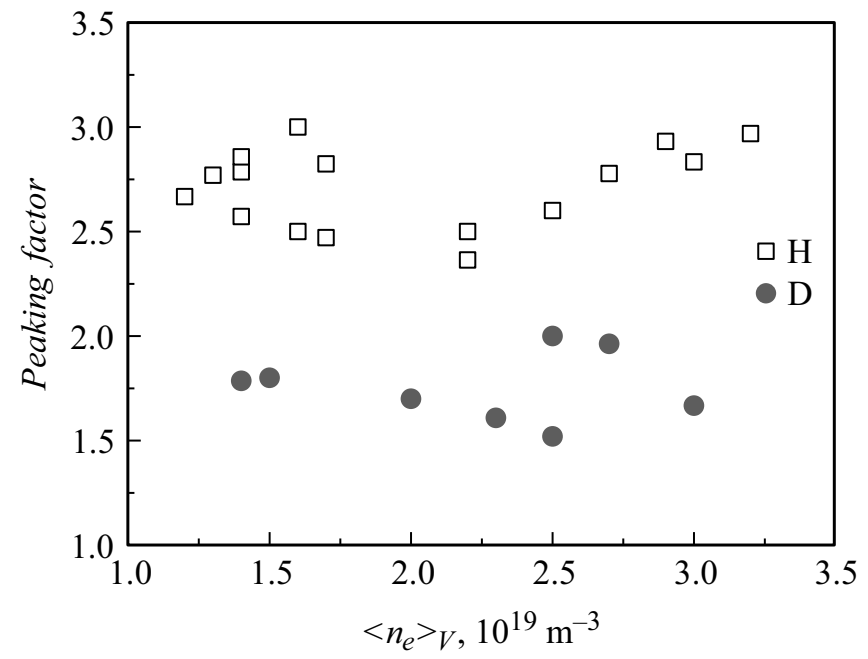

Рис. 3. Зависимость фактора пикируемости от средней плотности плазмы для двух серий разрядов.

для источника нейтронов, работающих по принципу „пучок плюс плазма“ $[14,15]$. В таком источнике нейтронный выход значительно зависит от температуры электронов, в то время как влияние плотности электронов проявляется значительно слабее. На токамаке
Глобус-М была проведена последняя экспериментальная кампания [16]. Последующие эксперименты на токамаке Глобус-М2 [17-19] (первая плазма на котором была получена в 2018 г. [20,21]) позволят продолжить исследования внутренних транспортных барьеров, возникающих в омических разрядах, в более широком диапазоне параметров плазмы.

\section{Благодарности}

Экспериментальные данные получены на УНУ „Сферический токамак Глобус-М“", входящей в состав ЦКП „Материаловедение и диагностика в передовых технологиях“. Работа со стандартными диагностиками была поддержана ФТИ им. А.Ф. Иоффе.

\section{Финансирование работы}

Моделирование процессов переноса с помощью численного кода ASTRA, а также все сопутствующие расчеты выполнены при финансовой поддержке Российского научного фонда (проект № 17-72-20076).

\section{Конфликт интересов}

Авторы заявляют, что у них нет конфликта интересов. 


\section{Список литературы}

[1] Гусев В.К., Голант В.Е., Гусаков Е.З., Дьяченко В.В., Ирзак М.А., Минаев В.Б., Мухин Е.Е., Новохацкий А.Н., Подушникова К.А., Раздобарин Г.Т., Сахаров Н.В., Трегубова Е.Н., Узлов В.С., Щербинин О.Н., Беляков В.А., Кавин А.А., Косцов Ю.А., Кузьмин Е.Г., Сойкин В.Ф., Кузнецов Е.А., Ягнов В.А. // ЖТФ. 1999. Т. 69. В. 9. C. $58-62$.

[2] Курскиев С.Г., Гусев В.К., Толстяков С.Ю., Березуикий А.А., Буланин В.В., Варфоломеев В.И., Кочергин М.М., Минаев В.Б., Мухин Е.Е., Патров М.И., Петров А.В., Петров Ю.В., Сахаров Н.В., Семёнов В.В., Яиин А.Ю., Хромов Н.А. // Письма в ЖТФ. 2011. Т. 37. B. 23. C. $82-90$.

[3] Telnova A.Yu., Kurskiev G.S., Bakharev N.N., Gusev V.K., Kiselev E.O., Khromov N.A., Minaev V.B., Miroshnikov I.V., Patrov M.I., Petrov Yu.V., Sakharov N.V., Sladkomedova A.D., Shchegolev P.B., Solokha V.V., Tokarev V.A., Tolstyakov S.Yu. // 45th EPS Conf. on plasma physics. Prague, Czech Republic, 2018. P. P5.1070.

[4] Андрейко М.В., Аскинази Л.Г., Голант В.Е., Жубр Н.А., Корнев В.А., Крикунов С.В., Лебедев С.В., Левин Л.С., Раздобарин. Г.Т., Рожсдественский В.В., Смирнов А.И., Тукачинский А.С., Ярошевич С.П. // Физика плазмы. 2000. T. 26. № 3. C. 209-216.

[5] Askinazi L.G., Bulanin V.V., Vildjunas M.I., Golant V.E., Gorokhov M.V., Kornev V.A., Krikunov S.V., Lebedev S.V., Petrov A.V., Rozhdestvensky V.V., Tukachinsky A.S., Zhubr N.A. // Plasma Phys. Control. Fusion. 2004. V. 46. P. A51-A60.

[6] Pereverzev G.V., Yushmanov P.N. // Max-Plank IPP report. 2002. P. 5-98.

[7] Lao L.L., John H.St., Stambaugh R.D., Kellman A.G., Pfeiffer W. // Nucl. Fusion. 1985. V. 25. P. 1611.

[8] Ware A.A. // Phys. Rev. Lett. 1970. V. 25. P. 15-17.

[9] Field A.R., Akers R.J., Applegate D.J., Brickley C., Carolan P.G., Challis C., Conway N.J., Cowley S.C., Cunningham G., Joiner N., Meyer H., Patel A., Roach C., Valovič M., Walsh M.J. and the MAST team // 20th IAEA Fusion Energy Conf. Vilamoura, Portugal, 2004. P. EX/P2-11.

[10] Kaye S.M., Levinton F.M., Stutman D., Tritz K., Yuh H., Bell M.G., Bell R.E., Domier C.W., Gates D., Horton W., Kim J., LeBlanc B.P., Luhmann N.C., Jr, Maingi R., Mazzucato E., Menard J.E., Mikkelsen D., Mueller D., Park H., Rewoldt G., Sabbagh S.A., Smith D.R., Wang W. // Nucl. Fusion. 2007. V. 47. P. 499-509.

[11] Тельнова А.Ю., Курскиев Г.С., Мирошников И.В., Авдеева Г.Ф., Бахарев Н.Н., Гусев В.К., Минаев В.Б., Мельник А.Д., Петров Ю.В., Сахаров Н.В., Чернышев Ф.В., Щёголев П.Б. // Письма в ЖТФ. 2018. Т. 44. В. 15. C. $100-110$.

[12] Курскиев Г.С., Сахаров Н.В., Щёголев П.Б., Бахарев Н.Н., Киселев Е.О., Авдеева Г.Ф., Гусев В.К., Ибляминова А.Д., Минаев В.Б., Мирошников И.В., Патров М.И., Петров Ю.В., Тельнова А.Ю., Толстяков С.Ю., Токарев В.А. // Вопр. атомной науки и техники. Сер. Термоядерный синтез. 2016. Т. 39. В. 4. С. 86-94.

[13] ITER Physics Basis // Nucl. Fusion. 1999. V. 39. P. 21752250.
[14] Menard J.E., Brown T., El-Guebaly L., Boyer M., Canik J., Colling B., Raman R., Wang Z., Zhai Y., Buxton P., Covele B., D'Angelo C., Davis A., Gerhardt S., Gryaznevich M., Harb M., Hender T.C., Kaye S., Kingham D., Kotschenreuther M., Mahajan S., Maingi R., Marriott E., Meier E.T., Mynsberge L., Neumeyer C., Ono M., Park J.-K., Sabbagh S.A., Soukhanovskii V., Valanju P., Woolley R. // Nucl. Fusion. 2016. V. 56. P. 106023.

[15] Gusev V.K., Bakharev N.N., Ber B.Ya., Bulanin V.V., Chernyshev F.V., Dyachenko V.V., Goncharov P.R., Gusakov E.Z., Iblyaminova A.D., Irzak M.A., Kaveeva E.G., Khitrov S.A., Khromov N.A., Kornev V.A., Kurskiev G.S., Melnik A.D., Minaev V.B., Mineev A.B., Mironov M.I., Novokhatsky A.N., Patrov M.I., Petrov A.V., Petrov Yu.V., Rozhansky V.A., Sakharov N.V., Saveliev A.N., Senichenkov I.Yu., Shchegolev P.B., Shcherbinin O.N., Tolstyakov S.Yu., Varfolomeev V.I., Voronin A.V., Yashin A.Yu. // Plasma Phys. Control. Fusion. 2016. V. 58. P. 014032.

[16] Bakharev N.N., Bulanin V.V., Chernyshev F.V., Gusev V.K., Khromov N.A., Kiselev E.O., Kurskiev G.S., Melnik A.D., Minaev V.B., Mironov M.I., Miroshnikov I.V., Patrov M.I., Petrov A.V., Petrov Yu.V., Sakharov N.V., Shchegolev P.B., Sladkomedova A.D., Solokha V.V., Telnova A.Yu., Tokarev V.A., Tolstyakov S.Yu., Yashin A.Yu. // Nucl. Fusion. 2018. V. 58. P. 126029.

[17] Gusev V.K., Azizov E.A., Alekseev A.B., Arneman A.F., Bakharev N.N., Belyakov V.A., Bender S.E., Bondarchuk E.N., Bulanin V.V., Bykov A.S., Chernyshev F.V., Chugunov I.N., Dyachenko V.V., Filatov O.G., Iblyaminova A.D., Irzak M.A., Kavin A.A., Kurskiev G.S., Khitrov S.A., Khromov N.A., Kornev V.A., Krasnov S.V., Kuznetsov E.A., Labusov A.N., Larionov M.M., Lobanov K.M., Malkov A.A., Melnik A.D., Minaev V.B., Mineev A.B., Mironov M.I., Miroshnikov I.V., Novokhatsky A.N., Ovsyannikov A.D., Panasenkov A.A., Patrov M.I., Petrov M.P., Petrov Yu.V., Rozhansky V.A., Rozhdestvensky V.V., Saveliev A.N., Sakharov N.V., Shchegolev P.B., Shcherbinin O.N., Senichenkov I.Yu., Sergeev V.Yu., Shevelev A.E., Stepanov A.Yu., Tanchuk V.N., Tolstyakov S.Yu., Varfolomeev V.I., Voronin A.V., Wagner F., Yagnov V.A., Yashin A.Yu., Zhilin E.G. // Nucl. Fusion. 2013. V. 53. P. 093013.

[18] Gusev V.K., Bakharev N.N., Belyakov V.A., Ber B.Ya., Bondarchuk E.N., Bulanin V.V., Bykov A.S., Chernyshev F.V., Demina E.V., Dyachenko V.V., Goncharov P.R., Gorodetsky A.E., Gusakov E.Z., Iblyaminova A.D., Ivanov A.A., Irzak M.A., Kaveeva E.G., Khitrov S.A., Khokhlov M.V., Khromov N.A., Kolmogorov V.V., Kornev V.A., Krasnov S.V., Kurskiev G.S., Labusov A.N., Lepikhov S.A., Litunovsky N.V., Mazul I.V., Melnik A.D., Mikov V.V., Minaev V.B., Mineev A.B., Mironov M.I., Miroshnikov I.V., Mukhin E.E., Novokhatsky A.N., Ovsyannikov A.D., Patrov M.I., Petrov A.V., Petrov Yu.V., Rozhansky V.A., Sakharov N.V., Saveliev A.N., Senichenkov I.Yu., Sergeev V.Yu., Shchegolev P.B., Shcherbinin O.N., Shikhovtsev I.V., Tanaev V.S., Tanchuk V.N., Tolstyakov S.Yu., Varfolomeev V.I., Vekshina E.O., Voronin A.V., Voskoboinikov S.P., Wagner F., Yashin A.Yu., Zadvitskiy G.V., Zakharov A.P., Zalavutdinov R.Kh., Zhilin E.G. // Nucl. Fusion. 2015. V. 55. P. 104016. 
[19] Minaev V.B., Gusev V.K., Sakharov N.V., Varfolomeev V.I., Bakharev N.N., Belyakov V.A., Bondarchuk E.N., Brunkov P.N., Chernyshev F.V., Davydenko V.I., Dyachenko V.V., Kavin A.A., Khitrov S.A., Khromov N.A., Kiselev E.O., Konovalov A.N., Kornev V.A., Kurskiev G.S., Labusov A.N., Melnik A.D., Mineev A.B., Mironov M.I., Miroshnikov I.V., Patrov M.I., Petrov Yu.V., Rozhansky V.A., Saveliev A.N., Senichenkov I.Yu., Shchegolev P.B., Shcherbinin O.N., Shikhovtsev I.V., Sladkomedova A.D., Solokha V.V., Tanchuk V.N., Telnova A.Yu., Tokarev V.A., Tolstyakov S.Yu., Zhilin E.G. // Nucl. Fusion. 2017. V. 57. P. 066047.

[20] Minaev V.B., Gusev V.K., Sakharov N.V., Petrov Yu.V., Varfolomeev V.I., Bakharev N.N., Bondarchuk E.N., Chernyshev F.V., Kavin A.A., Khromov N.A., Kurskiev G.S., Mineev A.B., Novokhatskii A.N., Oshuev K.Yu., Patrov M.I., Shchegolev P.B., Shevelev A.E., Sladkomedova A.D., Solokha V.V., Telnova A.Yu., Tokarev V.A., Tolstyakov S.Yu., Zhilin E.G. // 45th EPS Conf. on plasma physics. Prague, Czech Republic, 2018. P. P4.1065.

[21] Minaev V.B., Gusev V.K., Sakharov N.V., Petrov Yu.V., Varfolomeev V.I., Bakharev N.N., Belyakov V.A., Brunkov P.N., Bulanin V.V., Chernyshev F.V., Davydenko V.I., Dyachenko V.V., Kavin A.A., Khitrov S.A., Khromov N.A., Kiselev E.O., Konovalov A.N., Kornev V.A., Kurskiev G.S., Melnik A.D., Mineev A.B., Mironov M.I., Miroshnikov I.V., Novokhatskii A.N., Oshuev K.Yu., Patrov M.I., Petrov A.V., Rozhansky V.A., Saveliev A.N., Senichenkov I.Yu., Shchegolev P.B., Shikhovtsev I.V., Sladkomedova A.D., Solokha V.V., Sorokina D.S., Telnova A.Yu., Tokarev V.A., Tolstyakov S.Yu., Yashin A.Yu., Zhilin E.G. // J. Phys.: Conf. Ser. 2018. V. 1094. P. 012001. 